Mathematical Research Letters 1, 27-33 (1994)

\title{
OPERATOR VERSIONS OF THE VAN DER CORPUT LEMMA AND FOURIER INTEGRAL OPERATORS
}

\author{
D. H. Phong And E. M. Stein
}

\section{Introduction}

The purpose of this letter is to describe the regularity properties of certain oscillatory integral operators. As Fourier integral operators, their Lagrangians are not local graphs. Thus their regularity depends on the position of the Lagrangians within the ambient space. Except in the case of Whitney folds ([6][3][2][8][7][12][1]), this is however still only partially understood. Our results can be viewed as a step in a more systematic investigation of regularity in terms of a stratification of singularities.

We rely heavily on operator versions of the van der Corput lemma for non-degenerate phase functions whose Hessian can however be arbitrarily close to 0 . Such lemmas are surprisingly delicate, depending on the smoothness of the phase and the geometry of the support of the integrand. We formulate two versions below (Theorem 1 and Lemma 2). Our strategy is to find suitable decompositions of space into cubes, rectangles, or curved boxes where the operator van der Corput lemmas are applicable. Together with estimates on the size of the cross-sections of these boxes, the decomposition can be summed back to yield the desired regularity for oscillatory integrals and related Radon transforms.

In this letter we give only a brief sketch of the proofs. The full details will appear in [9], [10], and [11].

\section{Oscillatory integral operators}

The operators we consider are of the form

$$
T_{\lambda} f(x)=\int_{-\infty}^{\infty} e^{i \lambda S(x, y)} \chi(x, y) f(y) d y
$$

1991 Mathematics Subject Classification. Primary 42-xx, 42B20, 42B25, 35Sxx.

Key words and phrases. Lagrangians with folds, degenerate phases, uniform estimates, curved boxes, almost-orthogonality, Radon transforms..

Received October 29, 1993.

Supported in part by the National Science Foundation under grants DMS-92-04196 and DMS-91-04455.A01 
where $f \in C_{0}^{\infty}(\mathbb{R}), \chi \in C_{0}^{\infty}\left((0,1)^{2}\right)$, and $S(x, y)$ is a real smooth function of $(x, y) \in \mathbb{R}^{2}$. The phase $S(x, y)$ is said to be non-degenerate when $S_{x y}^{\prime \prime} \neq 0$ on $[0,1]^{2}$. It is well known that the norm $\left\|T_{\lambda}\right\|$ as an operator on $L^{2}(\mathbb{R})$ decays then at the rate $|\lambda|^{-1 / 2}$ as $|\lambda| \rightarrow \infty[4]$. The decay rate is however not uniform in $S$, and this is the key issue to which we turn. Thus we consider the class of $C^{\infty}$ phase functions satisfying the following conditions

$$
\begin{gathered}
\sum_{0 \leq p+q \leq n}\left|\frac{\partial^{p+q} S}{\partial x^{p} \partial y^{q}}(x, y)\right| \leq 1 \\
\left|\frac{\partial^{n} S}{\partial x \partial y^{n-1}}(x, y)\right|,\left|\frac{\partial^{n} S}{\partial x^{n-1} \partial y}(x, y)\right| \geq \mu>0
\end{gathered}
$$

on $[0,1]^{2}$ for some fixed integer $n \geq 2$. When $n=2$ we assume also that $\partial_{x}^{N-1} \partial_{y} S$ or $\partial_{x} \partial_{y}^{N-1} S$ does not vanish for some $N \geq 3$. An operator version of the van der Corput lemma uniform in the phases within this class is

Theorem 1. Under the conditions (2) the operator $T_{\lambda}$ has norm

$$
\left\|T_{\lambda}\right\| \leq C_{n}\left(\frac{|\lambda \mu|}{\log (1+|\lambda \mu|)}\right)^{-1 / n} \sum_{|\alpha| \leq 2} \sup _{[0,1]^{2}}\left|\partial^{\alpha} \chi\right|
$$

where $C_{n}$ is an absolute constant depending only on $n$ when $n \geq 3$, and on $N$ when $n=2$.

The cases $n=2$ and $n=3$ correspond respectively to the non-degenerate case and to the case of Lagrangians with two-sided Whitney fold singularities $([6][7])$. Estimates of the form (3) without the logarithmic factors hold then for individual phase functions, although even in these cases it is not known whether they hold with the degree of uniformity stated in Theorem 1. Under additional regularity assumptions on $S(x, y)$, we can treat asymmetric conditions on $S(x, y)$ as well as eliminate the logarithmic factors in (3). More precisely

Theorem 2. Let $S(x, y)$ be an analytic function of $(x, y)$ satisfying the conditions

$$
\frac{\partial^{p} S}{\partial x^{p-1} \partial y}(x, y) \neq 0, \frac{\partial^{q} S}{\partial x \partial y^{q-1}}(x, y) \neq 0
$$

for $(x, y)$ in the unit cube. Then

$$
\left\|T_{\lambda}\right\|=O\left(|\lambda|^{-\frac{p+q-4}{2(p q-p-q)}}\right) .
$$

For $C^{\infty}$ phases, the bound (4) with an additional factor $|\lambda|^{\epsilon}$ for any $\epsilon>0$ has been established by Seeger [12]. Of particular interest is the model case of phases which are homogeneous polynomials of degree $n$. There we have a complete characterization of the decay rate $\left\|T_{\lambda}\right\|=O\left(|\lambda|^{-1 / n}\right)$. 
Theorem 3. Let $S(x, y)$ be given by

$$
S(x, y)=\sum_{j=1}^{n-1} a_{j} x^{j} y^{n-j}
$$

Then a necessary and sufficient condition for the bound $\left\|T_{\lambda}\right\|=O\left(|\lambda|^{-1 / n}\right)$ to hold with an arbitrary choice of $C^{\infty}$ cut-off function $\chi(x, y)$ is the existence of a coefficient $a_{j} \neq 0$ with $j \leq n / 2$ and of a coefficient $a_{k} \neq 0$ with $k \geq n / 2$.

At the opposite end, we can achieve the optimal decay rate $\left\|T_{\lambda}\right\|=$ $O\left(|\lambda|^{-1 / 2}\right)$ for an arbitrary phase by inserting a damping factor in the amplitude. Such results play a key role in the study of $L^{p}-L^{q}$ estimates discussed below. For homogeneous polynomial phases, we have

Theorem 4. Let $S(x, y)$ be of the form (5), and consider the following oscillatory integral operator with damping factor $\sqrt{\left|S_{x y}^{\prime \prime}\right|}$ :

$$
U_{\lambda} f(x)=\int_{-\infty}^{\infty} e^{i \lambda S(x, y)} \sqrt{\left|\frac{\partial^{2} S}{\partial x \partial y}(x, y)\right|} \chi(x, y) f(y) d y
$$

Then for any coefficients $a_{j}, 1 \leq j \leq n-1$, we have $\left\|U_{\lambda}\right\|=O\left(|\lambda|^{-1 / 2}\right)$.

Estimates similar to those in Theorem 4 have been obtained in [5] for the translation-invariant case $S(x, y)=S(x-y)$.

Theorems 1-4 are established by decomposing the $(x, y)$-plane into boxes where the phase $S(x, y)$ is non-degenerate. To estimate the contribution of each box, we need an operator van der Corput lemma which is uniform in non-degenerate phase functions $S(x, y)$ with the same lower bound $\mu$ for the Hessian $S_{x y}^{\prime \prime}$.

For Theorem 1, such a van der Corput lemma is provided precisely by its $n=2$ subcase, which can be proved directly. When $n \geq 3$, the decomposition of $[0,1]^{2}$ is into squares, obtained by a stopping time process where $[0,1]^{2}$ is cut dyadically, and the dyadic cubes $Q_{\nu}$ satisfying either one of the following two conditions

$$
\begin{aligned}
& \min _{Q_{\nu}^{*}}\left|S_{x y}^{\prime \prime}\right| \geq \mu\left(\operatorname{diam} Q_{\nu}\right)^{n-2} \text { or } \\
& \min _{Q_{\nu}^{*}}\left|\partial_{x}^{n-2} \partial_{y} S\right|, \min _{Q_{\nu}^{*}}\left|\partial_{x} \partial_{y}^{n-2} S\right| \geq \mu\left(\operatorname{diam} Q_{\nu}\right)
\end{aligned}
$$

are retained. Here diam $Q_{\nu}$ and $Q_{\nu}^{*}$ denote respectively the diameter of $Q_{\nu}$ and the dilate of $Q_{\nu}$ by a factor of 16 . This decomposition resums to the 
desired estimate because the contributions of all squares of a fixed size are almost-orthogonal.

For Theorems 2-4, the van der Corput lemma required is more restrictive on the class of phase functions but allows amplitudes $\chi(x, y)$ supported on very general curved boxes. First, we introduce the class of polynomial type functions on $[0,1]$ as functions $F(x)$ such that

$$
\begin{aligned}
& \sup _{x \in I^{*}}|F(x)| \leq C \sup _{x \in I}|F(x)| \\
& \sup _{x \in I^{*}}\left|F^{\prime}(x)\right| \leq C|I|^{-1} \sup _{x \in I}|F(x)|
\end{aligned}
$$

where $I$ is any subinterval of $[0,1],|I|$ its length, $I^{*}$ its double in $[0,1]$, and $C$ is a constant independent of $I$. Functions of polynomial type arise via

Lemma 1. Let $F(x)$ be any $C^{N}$ function on $[0,1]$ which satisfies the following condition

$$
\sup _{x \in[0,1]}\left|F^{(N)}(x)\right| \leq C \inf _{x \in[0,1]}\left|F^{(N)}(x)\right| .
$$

Then $F(x)$ is of polynomial type.

The van der Corput lemma on curved boxes can now be stated as follows:

Lemma 2. Let $\varphi(x)$ be a monotone continuous function, and let $\mathcal{B}$ be the following curved box of thickness $\delta$ :

$$
\mathcal{B}=\{(x, y) ; \varphi(x)<y<\varphi(x)+\delta, 0<x<l\} .
$$

Let $T_{\lambda}$ be an operator of the form (1), with $\chi$ supported in $\mathcal{B}$ and satisfying the following estimates

$$
\sup _{x, y} \sum_{k=0}^{2}\left|\partial_{y}^{k} \chi(x, y)\right| \delta^{k} \leq 1 .
$$

Assume further that $S(x, y)$ is of polynomial type in $y$, and satisfies

$$
0<\mu \leq\left|\frac{\partial^{2} S}{\partial x \partial y}(x, y)\right| \leq A \mu .
$$

Then $\left\|T_{\lambda}\right\| \leq C_{A}\left(|\lambda \mu|^{-1 / 2}\right)$, with a constant $C_{A}$ independent of $\chi, \delta$, and $l$.

Lemma 2 is proved by covering the box $\mathcal{B}$ by almost-orthogonal rectangles $\mathcal{R}_{j}$ of the form (say, when $\varphi$ is increasing)

$$
\mathcal{R}_{j}=\left(x_{j}, x_{j+1}\right) \times\left(\varphi\left(x_{0}\right)+j \delta, \varphi\left(x_{0}\right)+(j+2) \delta\right)
$$


where $x_{0} \equiv 0$, and $x_{j}$ are defined by $\varphi\left(x_{j}\right)=\varphi\left(x_{0}\right)+j \delta$. We describe next the decomposition used for the proof of Theorem 2. It suffices to establish the theorem in a small neighborhood of an arbitrary point in $[0,1]^{2}$. Thus we may restrict ourselves to a small neighborhood of the origin, and in view of the Weierstrass preparation theorem, assume that $\partial^{2} S / \partial x \partial y(x, y)$ is of the form

$$
\frac{\partial^{2} S}{\partial x \partial y}(x, y)=U(x, y) \prod_{i=1}^{q-2}\left(y-r_{i}(x)\right)
$$

Here $U(x, y)$ is a smooth non-vanishing factor, and $r_{i}(x)$ are Puiseux series, i.e. power series in a fractional power of $x$. In particular, for each root $r_{i}(x)$, there is an $a_{i} \geq 0$ for which $r_{i}(x) \sim x^{a_{i}}$. For the sake of simplicity, we describe the partition of the region of non-degeneracy into curved boxes of $x, y$ space only in the case where all the roots are real, and $a_{1}<a_{2}<$ $\cdots<a_{q-2}$. The non-degeneracy region can be divided into sectors of the form $r^{a_{m}}(x)<y \leq 2 r^{a_{m}}(x)$ or $2 r^{a_{m+1}} \leq y<r^{a_{m}}(x)$. Assume further that $a_{m} \geq 1$, otherwise we reverse the roles of $x$ and $y$. Each of these sectors is decomposed in turn into curved boxes $\mathcal{B}_{j k}$ satisfying $x \sim 2^{-j}$ and $\left|y-r_{m}(x)\right| \sim 2^{-k}$. For example, for the first type of sector, we set

$$
T_{j k} f(x)=\int_{-\infty}^{\infty} e^{i \lambda S(x, y)} \phi_{j}(x) \phi_{k}\left(y-r_{m}(x)\right) \chi(x, y) f(y) d y
$$

where $\sum_{j} \phi_{j}=1$ is a dyadic partition of unity on $\mathbb{R}$ with $\phi_{j}(x)=0$ outside of $2^{-j-1} \leq x \leq 2^{-j+1}$. The second type of sector is treated in the same way with obvious modifications. Lemma 2 applies to $T_{j k}$, since $r_{m}(x)$ is a Puiseux series and admits only a finite number of intervals of monotonicity. Together with estimates on $\left\|T_{j k}\right\|$ resulting from the size of the cross-sections of $\mathcal{B}_{j k}$, the double series $\left\|T_{j k}\right\|$ can be resummed to produce the estimates stated in Theorem 2 .

Finally Theorems 3 and 4 are established by superposing a decomposition similar to the above decomposition $\left\{\mathcal{B}_{j k}\right\}$ of the sectors of nondegeneracy with a further dyadic decomposition $y \sim 2^{-l}$. The necessary and sufficient condition stated in Theorem 3 is needed to control the contributions of the boxes which are very close to either one of the two coordinate axes.

\section{Radon transforms}

The above estimates correspond to regularity properties for certain degenerate Fourier integral operators. More precisely, given a $(n-1)$ vectorvalued phase function

$$
S(x, y)=\left(S_{1}(x, y), \cdots, S_{n-1}(x, y)\right)
$$


we can define the family of curves $\gamma_{t, x}$ in $\mathbb{R}^{n}$ by $\gamma_{(t, x)}=\left\{(s, y) \in \mathbb{R}^{n-1} \times\right.$ $\mathbb{R} ; s=t+S(x, y)\}$. Let $d \mu_{(t, x)} \equiv \delta(t-s+S(x, y)) \chi(x, y) d y$ be a smooth compactly supported density on $\gamma_{(t, x)}$. Then the Radon transform associated to the family of curves $\gamma$ is defined by

$$
\begin{aligned}
R f(t, x) & =\int_{\gamma_{(t, x)}} f d \mu_{(t, x)} \\
& =\int_{-\infty}^{\infty} e^{i<t, \lambda>}\left(T_{\lambda} \hat{f}(\cdot, \lambda)\right) d \lambda
\end{aligned}
$$

where $\hat{f}(y, \lambda)$ is the partial Fourier transform of $f$ with respect to $t$. The operator $R$ is a Fourier integral operator with Lagrangian $\Lambda$ given by the normal bundle $N(\mathcal{C})$ of the incidence manifold $\mathcal{C}=\{(t, x ; s, y) ;(s, y) \in$ $\left.\gamma_{(t, x)}\right\}$. In dimension $n=2$, the Lagrangian $\Lambda$ is locally the (reflection of the) graph of a canonical transformation when $\frac{\partial^{2} S}{\partial x \partial y} \neq 0$. In dimension $n>2$, dimension counting shows that both projections $\pi_{L}$ and $\pi_{R}$ from $T^{*}\left(\mathbb{R}_{(t, x)}^{n}\right) \times T^{*}\left(\mathbb{R}_{(s, y)}^{n}\right)$ to $T^{*}\left(\mathbb{R}_{(t, x)}^{n}\right)$ and $T^{*}\left(\mathbb{R}_{(s, y)}^{n}\right)$ must have singularities. The Thom-Boardman classification of singularities suggests describing $\pi_{L}$ and $\pi_{R}$ in terms of the following varieties:

$$
\begin{aligned}
& \Sigma_{L}^{(k)}=\Lambda \cap\left\{\left\langle\lambda, \frac{\partial^{m} S}{\partial x \partial y^{m-1}}\right\rangle=0,2 \leq m \leq k\right\} \\
& \Sigma_{R}^{(k)}=\Lambda \cap\left\{\left\langle\lambda, \frac{\partial^{m} S}{\partial x^{m-1} \partial y}\right\rangle=0,2 \leq m \leq k\right\}
\end{aligned}
$$

Here we have parametrized $\Lambda$ by

$$
\Lambda=\left\{\left(t, \lambda, x,<\lambda, S_{x}^{\prime}>; s,-\lambda, y,<\lambda, S_{y}^{\prime}>\right) ;(t, x ; s, y) \in \mathcal{C}, \lambda \in \mathbb{R}^{n-1} \backslash 0\right\}
$$

A natural generic condition for families of curves in $\mathbb{R}^{n}$ is that $\Sigma_{L}^{(n)}$ and $\Sigma_{R}^{(n)}$ be both empty. For translation invariant families, i.e. $S(x, y) \equiv S(x-$ $y)$, this reduces to the condition of non-vanishing torsion for the curve $t+S(x)=0$. Thus we shall say that the variable family of curves has (two-sided) torsion when this condition is verified.

Theorem 5. Let $S(x, y)$ define a smooth family of curves in $\mathbb{R}^{n}$ with nonvanishing torsion in the above sense, and let $R$ be the corresponding Radon transform. Then

(a) The operator $R$ is smoothing from $H_{(s)}$ to $H_{\left(s+\frac{1}{n}-\epsilon\right)}$ for any $\epsilon>0$;

(b) When the family of curves is analytic, $R$ is smoothing from $H_{(s)}$ to $H_{\left(s+\frac{1}{n}\right)}$; 
(c) In dimension $n=2$, when the phase $S(x, y)$ is given by a homogeneous polynomial of the form (5), the operator $R$ is bounded from $L^{p}\left(\mathbb{R}^{2}\right)$ to $L^{q}\left(\mathbb{R}^{2}\right)$ when $(1 / p, 1 / q)$ is in the intersection of the halfplane $(1 / p)-(1 / q) \leq 1 /(n+1)$ with the convex hull of the segment $0<1 / p=1 / q<1$ and the point $(2 / 3,1 / 3)$.

\section{References}

1. A. Greenleaf and A. Seeger, Fourier integral operators with fold singularities, preprint, 1993.

2. A. Greenleaf and G. Uhlmann, Composition for some singular Fourier integral operators and estimates for the restricted X-ray transform, I, Ann. Inst. Fourier 40 (1990), 443-466; II, Duke Math. J. 64 (1991), 415-444.

3. V. Guillemin, Cosmology in $2+1$ dimensions, cyclic models, and deformations, Princeton University Press, 1990.

4. L. Hörmander, Oscillatory integrals and multipliers on $F L^{p}$, Arkiv. Mat. 11 (1973), $1-11$.

5. C. Kenig, G. Ponce, and L. Vega, Oscillatory integrals and regularity of dispersive equations, Indiana Math. J. 40 (1991), 33-69.

6. R. Melrose and M. Taylor, Near peak scattering and the corrected Kirkhhoff approximation for a convex obstacle, Advances Math. 44 (1985), 242-315.

7. Y. Pan and C. Sogge, Oscillatory integrals associated to canonical folding relations, Colloquium Mathematicum 40 (1990), 413-419.

8. D. H. Phong and E. M. Stein, Radon transforms and torsion, Int. Math. Res. Notices 4 (1991), 49-60.

9. __ Models of degenerate Fourier integral operators and Radon transforms, Annals of Math. (to appear).

10. appear).

11. , in preparation.

12. A. Seeger, Degenerate Fourier integral operators in the plane (to appear).

Departiment of Mathematics, Columbia University, New York, Ny 10027

E-mail address: phong@shire.math.columbia.edu

Department of Mathematics, Princeton Univeritit, Princeton, NJ 08544

E-mail address: stein@math.princeton.edu 